\title{
Jmjd3 is essential for the epigenetic modulation of microglia phenotypes in the immune pathogenesis of Parkinson's disease
}

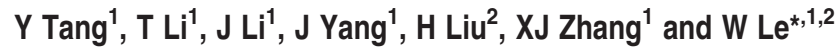

Classical activation (M1 phenotype) and alternative activation (M2 phenotype) are the two polars of microglial activation states that can produce either detrimental or beneficial effects in the central nervous system (CNS). Harnessing the beneficial properties of microglia cells by modulating their polarization states provides great potential for the treatment of Parkinson's disease (PD). However, the epigenetic mechanism that regulates microglia polarization remains elusive. Here, we reported that histone H3K27me3 demethylase Jumonji domain containing 3 (Jmjd3) was essential for M2 microglia polarization. Suppression of Jmjd3 in N9 microglia inhibited M2 polarization and simultaneously exaggerated M1 microglial inflammatory responses, which led to extensive neuron death in vitro. We also observed that the suppression of Jmjd3 in the substantia nigra (SN) in vivo dramatically caused microglial overactivation and exacerbated dopamine (DA) neuron death in 1-methyl-4-phenyl-1, 2, 3, 6-tetrahydropyridine (MPTP)-intoxicated mouse model of PD. Moreover, we showed that the Jmjd3 level was lower in the midbrain of aged mice, which was accompanied by an elevated level of H3K27me3 and an increased ratio of M1 to M2 markers, suggesting that aging is an important factor in switching the microglia phenotypes. Overall, our studies indicate that Jmjd3 is able to enhance the polarization of M2 microglia by modifying histone H3K27me3, and therefore it has a pivotal role in the switch of microglia phenotypes that may contribute to the immune pathogenesis of PD.

Cell Death and Differentiation (2014) 21, 369-380; doi:10.1038/cdd.2013.159; published online 8 November 2013

Parkinson's disease (PD) is the second most common neurodegenerative disorder, which is characterized by selective loss of dopamine (DA) neurons, Lewy body (LB) formation, and excessive reactive microgliosis. ${ }^{1,2}$ Overwhelmingly activated microglia are present in the vicinity of the degenerating neurons in the substantia nigra (SN) of PD patients. $^{3}$

Microglial activation can be classified into two major phenotypes defined as 'classical activation' (also termed M1 phenotype or overactivated phenotype) and 'alternative activation' (M2 phenotype). ${ }^{4,5}$ M1 microglia polarization is associated with the production and release of multiple proinflammatory cytokines. ${ }^{6-10}$ The released factors generally act in tissue defense and promote the destruction of pathogens. ${ }^{11,12}$ However, overactivated or dysregulated microglia are constantly involved in the pathogenesis of PD and serve to amplify neuronal damage caused by pathological stimuli and toxins, which in turn, induces more widespread damage to the neighboring neurons. ${ }^{13,14}$ In contrast to the M1 phenotype, M2 microglia execute an anti-inflammatory effect and promote wound healing and tissue repair. ${ }^{15,16}$ The major anti-inflammatory cytokines, such as interleukin-4 (IL-4), IL-13, IL-10 and transforming growth factor- $\beta$ (TGF- $\beta$ ), initiate the alleviation of pro-inflammatory responses and enhance the expression of genes that are involved in tissue recovery and repair. This resolution state is critical in chronic neuroinflammation-related diseases including PD.

In different pathogenetic stages of PD, microglial activation states may change throughout a pathologic process. Although microglial activation is necessary and crucial for host defense and neuron survival, persistent microglial overactivation may finally result in deleterious consequences. ${ }^{17}$ For the future treatment of PD, it will be interesting to know whether there is an effective strategy to halt the toxic phase of M1 microglia polarization and restore tissue homeostasis by switching the microglia phenotypes or enhancing the beneficial effects of M2 microglia, a notion of which is supported by a recent study in the treatment of experimental autoimmune neuritis disease. ${ }^{18}$

Epigenetic is a regulator of gene expression by modifying histone tails or changing DNA methylation. ${ }^{19}$ Increasing studies have already shed light on the relationship between

\footnotetext{
${ }^{1}$ Institute of Health Sciences, Shanghai Institutes for Biological Sciences, Chinese Academy of Sciences, Shanghai, China and ${ }^{2}$ Institute of Neurology, Ruijin Hospital, Shanghai Jiao Tong University School of Medicine, Shanghai, China

*Corresponding author: W Le, Institute of Health Sciences, Shanghai Institutes for Biological Sciences, Chinese Academy of Sciences, Shanghai, China. Tel: +86 2164370045 Ext. 611201; Fax: +86 21 54669084; E-mail: wdle@sibs.ac.cn

Keywords: Parkinson's disease; microglia polarization; histone H3K27me3 demethylase; Jmjd3; aging; neuron degeneration

Abbreviations: PD, Parkinson's disease; LB, Lewy body; DA neuron, dopamine neuron; SN, substantia nigra; Jmjd3, Jumonji domain containing 3; UTX, Ubiquitously transcribed tetratricopeptide repeat, $\mathrm{X}$ chromosome; CM, conditioned medium; PM, primary microglia; VM, ventral mesencephalic; IL, interleukin; TGF- $\beta$, transforming growth factor- $\beta$; TNF- $\alpha$, tumor necrosis factor- $\alpha$; IFN $\gamma$, interferon- $\gamma$; MPTP, 1-methyl-4-phenyl-1, 2, 3, 6-tetrahydropyridine; TH, Tyrosine hydroxylase; BDNF, brainderived neurotrophic factor; GDNF, glial cell-derived neurotrophic factor; iNOS, inducible nitric oxide synthase; Ezh2, enhancer of zeste homolog 2; UPS, ubiquitinproteasome system

Received 26.5.13; revised 26.9.13; accepted 01.10.13; Edited by B Dynlacht; published online 08.11.13
} 
epigenetic alterations and neurodegenerative diseases. ${ }^{20}$ In the present study, we demonstrate that histone H3K27me3 demethylase Jumonji domain containing 3 (Jmjd3) is essential for M2 microglia polarization. Suppression of Jmjd3 switches microglia from the beneficial M2 phenotype into the detrimental M1 phenotype, which finally accelerates DA neuron death.

\section{Results}

Microglia can be polarized into different functional phenotypes. To verify whether microglia can be polarized into detrimental M1 and beneficial M2 phenotypes, we employed lipopolysaccharide/interferon- $\gamma$ (LPS/IFN $\gamma$ ) as M1 triggers or IL-4/IL-13 cytokines (IL-4/IL-13) as M2 triggers to stimulate $\mathrm{N} 9$ microglia. We found that LPS/IFN $\gamma$ treatment enhanced the mRNA levels of most M1 markers, whereas it inhibited the mRNA levels of M2 markers (Supplementary Figure S1A). On the contrary, treatment with IL-4/IL-13 dramatically increased the mRNA levels of M2 markers, whereas compromised the expression of various M1 markers (Supplementary Figure S1A).

We then cultured the N2A neurons with conditioned medium (CM) from N9 microglia or Raw264.7 macrophages, which were stimulated with $\mathrm{M} 1$ or $\mathrm{M} 2$ triggers. We observed that cultures with $\mathrm{CM}$ from cells treated with $\mathrm{M} 1$ triggers (M1-CM) led to greater neuron death, whereas CM cocktails from cells treated with combined M1 and M2 triggers (M12$\mathrm{CM}$ ) could reverse the neurotoxicity produced by the M1-CM (Supplementary Figures S1B and C). This suggests that IL-4/ IL-13 treatment can antagonize M1 classical activation. Nitric oxide (NO), a reactive radical catalyzed by inducible nitric oxide synthase (iNOS), which was reported to have a role in the PD pathogenesis, ${ }^{6}$ can cause neuron death. To examine the level of released NO from N9 cells, we quantitatively measured the content of nitrate in the $\mathrm{CM}$ and found that it was significantly elevated in M1 microglia but was suppressed by the addition of M2 cytokines (Supplementary Figure S1D).

We also cultured primary ventral mesencephalic (VM) neurons with $\mathrm{M} 1-\mathrm{CM}$ or $\mathrm{M} 2-\mathrm{CM}$ to observe the effects of $\mathrm{CM}$ on axon degeneration. We found that cultures treated with $\mathrm{M} 1-\mathrm{CM}$ but not control-CM or M2-CM resulted in fragmented axons of the DA neurons (Supplementary Figure S1E). Overall, we demonstrate that microglia can be polarized into different functional phenotypes with either detrimental or beneficial effects to neurons.

Histone H3K27me3 demethylase Jmjd3 is induced in IL-4-treated microglia. We next asked whether epigenetic mechanism was involved in M2 microglia polarization. As the demethylation of histone was discovered in recent years and emerged as a critical factor contributing to various human diseases, we focused on the histone demethylases that may regulate this process. We treated primary microglia $(\mathrm{PM})$ with IL-4 and screened for potential candidates. We found that Jmjd3 was induced upon IL-4 treatment in PM cells, as well as in N9 microglia and Raw264.7 macrophages (Figure 1a). Whereas in the N2A and SHSY-5Y neurons as well as in the primary astrocytes, the mRNA levels of Jmjd3 were not altered by IL-4 treatment, indicating that Jmjd3 augmentation
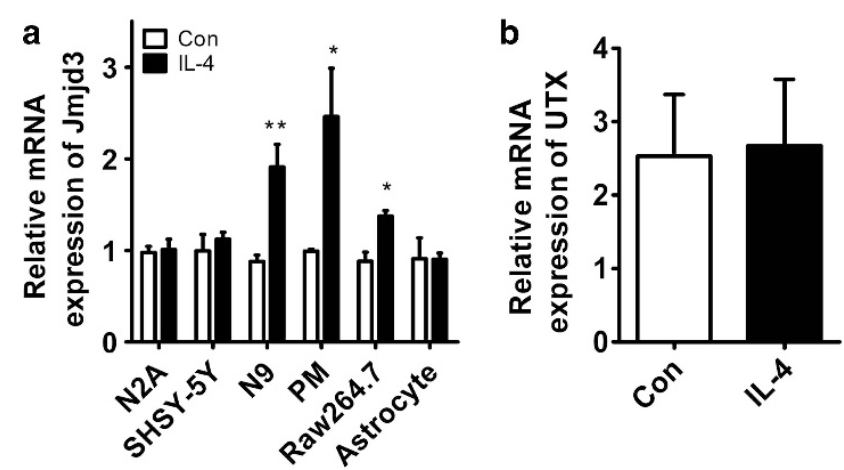

Figure 1 Jmjd3, not its homolog UTX, responds to IL-4 treatment in microglia. (a) Different cells including N2A, SHSY-5Y, N9 microglia, PM, Raw264.7 macrophages and primary astrocytes were stimulated with IL-4 $(10 \mathrm{ng} / \mathrm{ml})$ for $24 \mathrm{~h}$. The mRNA expression of Jmjd3 was then detected using real-time PCR. (b) The mRNA level of Jmjd3 homolog, UTX, was also determined using real-time PCR in the IL-4-treated N9 cells. ${ }^{*} P<0.05,{ }^{\star *} P<0.01$

is microglia-specific (Figure 1a). Jmjd3 is a histone H3K27me3 demethylase belonging to ubiquitously transcribed tetratricopeptide repeat, $\mathrm{X}$ chromosome (UTX) subfamily of demethylases. ${ }^{21}$ We then determined the expression of UTX, which is a homolog of Jmjd3. We found that UTX was not altered upon IL-4 treatment, suggesting that Jmjd3 not UTX participates in IL-4-elicited M2 polarization (Figure 1b).

Jmjd3 is essential for M2 microglia polarization depending on its demethylase activity. To investigate the role of Jmjd3 in M2 microglia polarization, we employed both loss-of-function and gain-of-function strategies. We generated stable Jmjd3-knockdown (kdJmjd3) N9 cells by lentivirus infection. Downregulation of Jmjd3 in N9 cells increased the H3K27me3 level without altering the UTX expression (Figures 2a and b). Moreover, we observed that microglia proliferation was not affected upon Jmjd3 suppression (Figure 2c).

We then stimulated N9 cells with IL-4 and detected the M2 gene expression using real-time PCR. Interestingly, we found that, even without IL-4 stimulation, the M2 markers such as Arginase1 and CD206 were significantly decreased when Jmjd3 was knocked down (Figures 2d and e). Accordingly, the increased expression of Arginase1, CD206 and IRF4 initiated by IL-4 treatment was significantly repressed in the knockdown cells (Figures $2 \mathrm{~d}-\mathrm{f}$ ). PPAR $\gamma$, a classical transcription factor that promotes M2 polarization, however, was not affected by Jmjd3 knockdown (Figure 2g). Similarly, Arginase1 expression was also compromised in Jmjd3-knockdown PM cells (Figure $2 \mathrm{~h}$ ).

In the study of M2 phenotype in macrophages, three subtypes (M2a, M2b and M2c) could be further divided in responding to different sets of cytokines. ${ }^{22}$ Specifically, IL-4 and IL-13 initiated the M2a subtypes, whereas IL-10 elicited the M2c subtype. ${ }^{22}$ Thus, we treated N9 microglia with IL-10 and found that Arginase1 expression was upregulated, whereas suppression of Jmjd3 could inhibit the increase in Arginase1 expression (Supplementary Figure S2). Overall, these results show that Jmjd3 is required for M2 microglia polarization. 
a

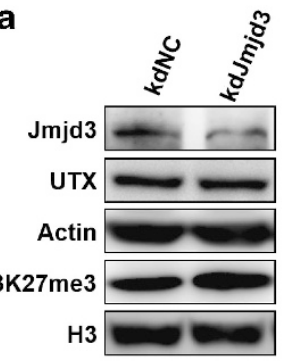

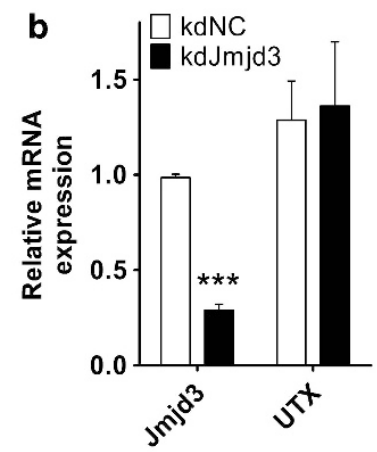

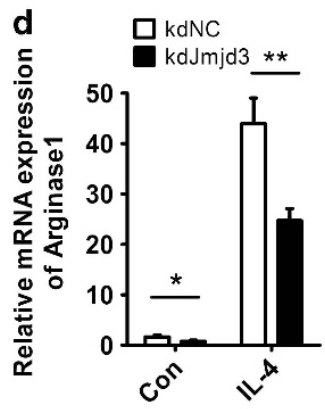

e

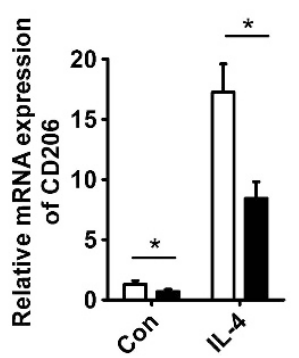

f

C

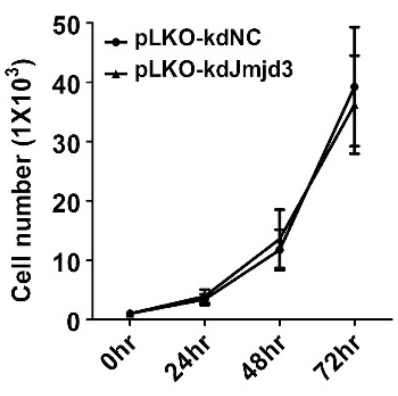

g

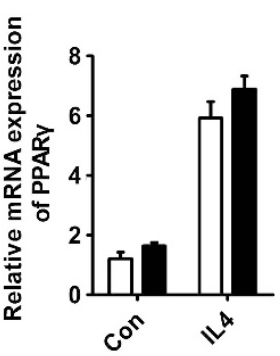

h

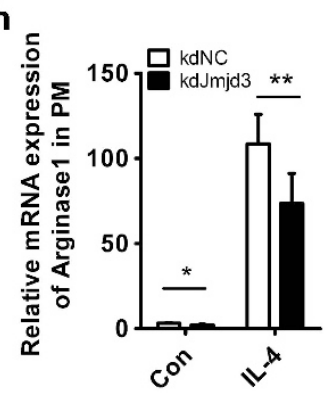

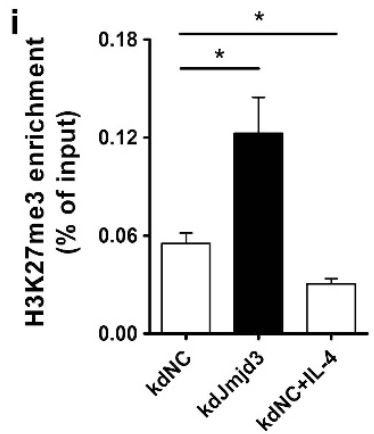

k

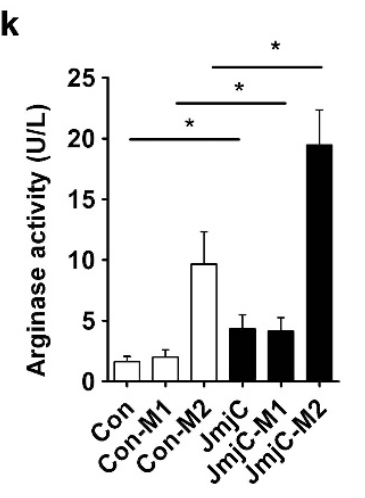

I

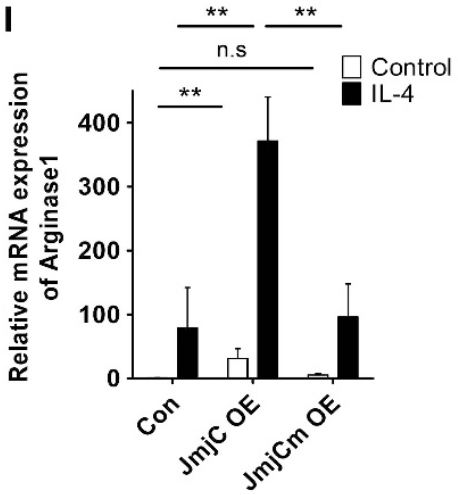

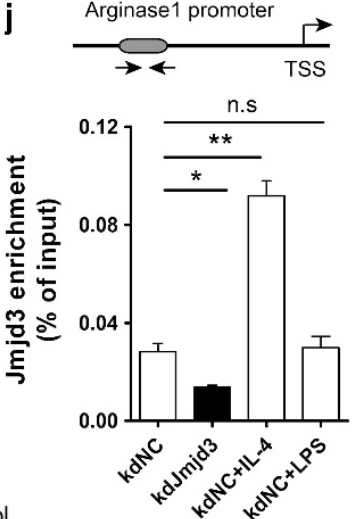

m

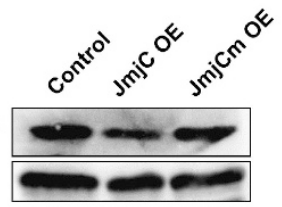

Figure $2 \mathrm{Jmjd} 3$ is essential for M2 microglia polarization depending on its demethylase activity. (a) Jmjd3 expression in the N9 microglia was knocked down by infection with pLKO-kdNC and pLKO-kdJmid3 lentiviruses. The protein expression of Jmid3, UTX and H3K27me3 was detected using immunoblotting. (b) Real-time PCR was performed to determine the mRNA level of Jmjd3 and UTX in the infected N9 cells. (c) Lentivirus-infected N9 cells were plated in a 96-well plate and the cell viability was measured using the MTT assay. $(\mathbf{d}-\mathrm{g})$ The Jmjd3-knockdown N9 cells were stimulated with IL-4 $(10 \mathrm{ng} / \mathrm{ml})$ for $24 \mathrm{~h}$. The mRNA expression of related M2 markers was then determined using real-time PCR. (h) Jmjd3 expression was knocked down in PM cells followed by treatment with IL-4 (10 ng/ml) for $24 \mathrm{~h}$. Arginase1 expression was then assessed using real-time PCR. (i and j) Lentivirus-infected N9 cells were treated with IL-4 $(10 \mathrm{ng} / \mathrm{ml})$ or LPS (100 ng/ml) for $24 \mathrm{~h}$ and immunoprecipitated with anti-H3K27me3 (i) and anti-Jmjd3 (j) antibodies. ChIP DNA levels were analyzed using semi-quantitative PCR and normalized to the levels of input DNA. (k) The JmjC domain was introduced into N9 cells by lentivirus (pLVX-JmjC) infection followed by treatment with $\mathrm{M} 1$ and $\mathrm{M} 2$ triggers, respectively, for $24 \mathrm{~h}$. The arginase activity was then measured. (I) N9 cells were infected with pLVX-JmjC- and pLVX-JmjCm-overexpressed lentiviruses and treated with IL-4 (10 ng/ml) for $24 \mathrm{~h}$. The mRNA expression of Arginase1 was then assessed using real-time PCR. (m) The H3K27me3 level in the infected N9 cells was determined using the immunoblotting assay. ${ }^{\star} P<0.05,{ }^{* \star} P<0.01,{ }^{* \star \star} P<0.001$, n.s, not significant 
We then performed chromatin immunoprecipitation (ChIP) to assess the H3K27me3 and Jmjd3 recruitment to the promoter of Arginase1. We found that H3K27me3 recruitment was significantly increased in Jmjd3-knockdown N9 cells but was significantly decreased upon IL-4 treatment (Figure 2i). On the contrary, the signal for Jmjd3 binding to the Arginase1 promoter was greatly reduced in the knockdown cells, whereas it was enhanced after IL-4 treatment but not after LPS treatment (Figure 2j). These results suggest that Arginase1 is a direct target of Jmjd3-mediated M2 polarization.

$\mathrm{JmjC}$ domain is the catalytic domain of Jmjd3, which is critical in demethylating histone H3K27me3 to H3K27 me2/me. ${ }^{21}$ We then overexpressed the JmjC domain in N9 cells and found that it significantly increased the arginase activity independent of LPS or IL-4 treatment (Figure $2 \mathrm{k}$ ). This suggests that Jmjd3 is sufficient for generating M2 microglia phenotype. We also examined the mRNA level of Arginase1 using real-time PCR. Similarly, IL-4-induced Arginase1 expression was dramatically enhanced by JmjC overexpression (Figure 2l).

To test the role of demethylase activity in this process, we generated a JmjC mutant $(\mathrm{H} 1388 \mathrm{~A})$, in which one amino acid in the $\mathrm{JmjC}$ domain involved in $\mathrm{Fe}^{2+}$ binding was mutated so that the demethylase activity was diminished. ${ }^{21}$ Indeed, when this mutant was introduced into microglia, no reduction in the H3K27me3 level was observed, contrary to the globally decreased H3K27me3 by JmjC overexpression (Figure 2m). We also found that the elevated Arginase1 expression induced by JmjC overexpression was dismissed in the JmjCm-overexpressed cells, demonstrating that Jmjd3 modulates M2 microglia polarization through its effect on its demethylase activity (Figure 2l).

\section{Suppression of Jmjd3 leads to enhanced M1 microglia} polarization. As M2 microglia are able to antagonize the effects of M1 classical activation, it is interesting to assess M1 polarization upon Jmjd3 suppression. We thus determined the levels of several key pro-inflammatory factors that are expressed in microglia. We found that the expression levels of both iNOS and IL-1 $\beta$ were greatly enhanced when Jmjd3 was knocked down (Figure 3a). In line with the immunoblotting result, the level of $\mathrm{NO}$ in the $\mathrm{CM}$ was accordingly elevated when Jmjd3 was repressed (Figure 3b). Moreover, ELISA measurement of the CM showed a significant higher IL-6 level upon Jmjd3 knockdown (Figure 3c). Whereas the tumor necrosis factor- $\alpha$ (TNF- $\alpha$ ) level in the CM was unexpectedly downregulated, its response to LPS insult was not compromised (Figure 3d). This may be because of diverse transcriptional pathways that activate pro-inflammatory process.

Pro-inflammatory stimuli and stress conditions rapidly activate the transcription factor $\mathrm{NF}_{\kappa} \mathrm{B}$, which subsequently induces transcription from several genes, including inflammatory cytokines and chemokines. ${ }^{23}$ Suppression of $\mathrm{NF} \kappa \mathrm{B}$ attenuates microglial activation, thereby rendering the neuroprotection. ${ }^{24,25}$ We found that, in the Jmjd3-knockdown N9 cells, the phosphorylation of $\mathrm{NF}_{\kappa} \mathrm{B}$ was significantly enhanced, which might contribute to amplify the pro-inflammation (Figures 3e and f). Collectively, suppression of Jmjd3 compromises M2 microglia polarization and accordingly leads to exaggerated M1 microglial inflammatory responses.

Suppression of Jmjd3 in microglia is detrimental to cultured neurons. We then asked whether the switch of microglia from M2 to M1 phenotype would cause greater neuron death. To address this question, we cultured N2A neurons with $\mathrm{CM}$ collected from control, M1 or M2 microglia cultures. The incubation of M1-CM from N9 cells caused significant neuron death, whereas M2-CM could effectively reverse it (Figure 4a). However, when Jmjd3 was suppressed in the microglia, M2-CM was not able to rescue the neuron death induced by M1-CM (Figure 4a), demonstrating that the M2 cytokine treatment cannot antagonize the M1 classical inflammation without Jmjd3.

Interestingly, CM from Jmjd3-knockdown N9 cells without any treatment (kdJmjd3-CM) was sufficient to induce $\sim 50 \%$ neuron death in the cultures (Figure $4 \mathrm{a}$ ). The higher levels of cleaved PARP and cleaved caspase 3 of the cultured N2A neurons were induced by culturing with kdJmjd3-CM, suggesting that apoptotic pathways may participate in the neuron death (Figures $4 b$ and $c$ ). Similarly, we also cultured primary VM neurons with kdNC-CM or kdJmjd3-CM, and we found that kdJmjd3-CM was much more detrimental to VM neurons especially the DA neurons (Figures $4 d$ and e).

In addition, we cultured N2A neurons with $\mathrm{CM}$ from either Jmjd3-knockdown N2A or SHSY-5Y neurons, and we found that the neuron survival was not affected, suggesting that Jmjd3 knockdown in neurons cannot produce cell-autonomous toxic effects (Figures $4 \mathrm{f}$ and $\mathrm{g}$ ). Taken together, these results indicate that the suppression of Jmjd3 in microglia is detrimental to cultured neurons and accelerates neuronal apoptosis.

Arginase1, the downstream target of Jmjd3, prevents neuron death through combating with iNOS. Arginase1, one of the direct targets of Jmjd3, is considered as a typical M2 marker and critical neuron-survival molecule. One of the potential mechanisms for Arginase1 involvement in inflammation is its competition with iNOS, as both Arginase1 and iNOS use the same substrate, L-arginine. ${ }^{26}$ We aimed to determine whether Jmjd3 regulates the balance between iNOS and Arginase1. To this end, we employed an Arginase1 inhibitor (nor-NOHA) and an iNOS inhibitor (L-NAME) in the cultures of N9 microglia. We found that norNOHA-treated PM produced high level of NO that can be reversed by L-NAME addition (Supplementary Figure S3).

We then downregulated the expression of Jmjd3 or Arginase1 in the N9 microglia and then treated with LPS, nor-NOHA and L-NAME in different combinations (Figures $2 b$ and $5 \mathrm{a}$ ). We found that both Jmjd3 and Arginase1 suppressions led to elevated NO in the CM. Interestingly, the changing profile of NO levels in the kdJmjd3 cells was very similar to that in Arginase1 knockdown (kdArginase1) cells (Figure 5b). Introducing full-length Arginase1 into kdJmjd3 cells could greatly reverse the NO production (Figure $5 \mathrm{~b}$ ). These results suggest that NO elevation caused by Jmjd3 knockdown may be partly due to Arginase1 suppression.

Extracellular arginine is transported into cells to regulate the intracellular NO production. ${ }^{27}$ We thus cultured N9 cells with 
a
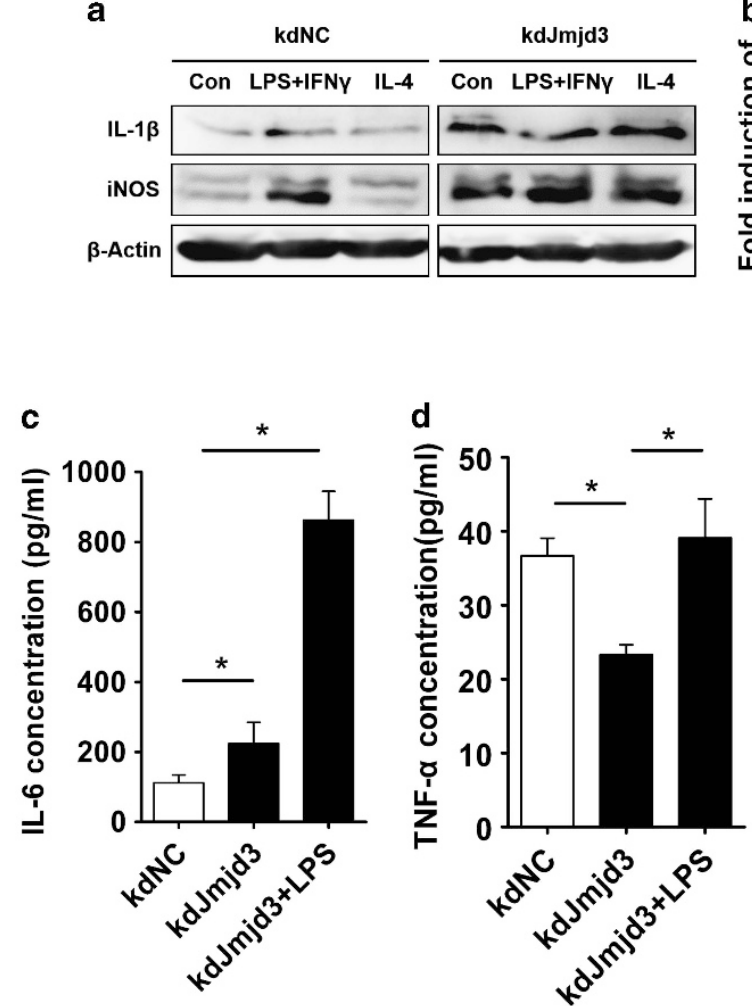
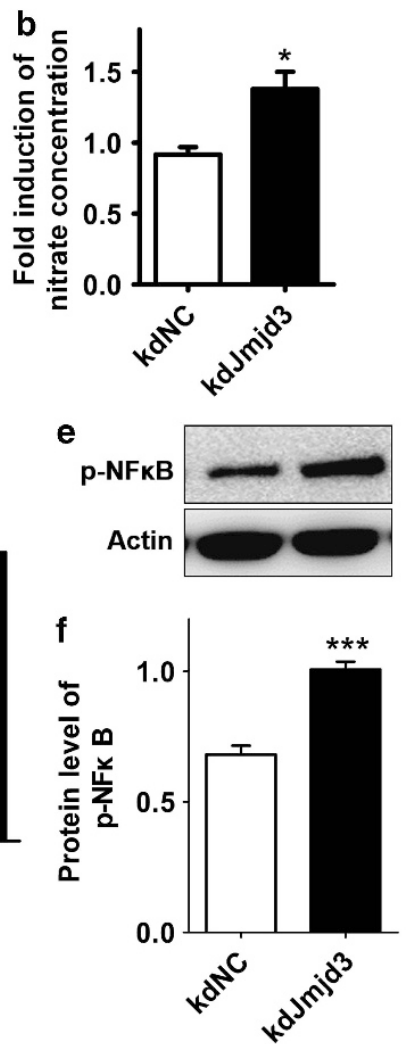

Figure 3 Knockdown of Jmjd3 enhances M1 microglia polarization. (a) N9 cells were infected with pLKO-kdNC and pLKO-kdJmjd3 lentiviruses followed by treatment with M1 and M2 triggers, respectively, for $24 \mathrm{~h}$. Proteins were then isolated to detect the expression of pro-inflammatory factors IL-1 $\beta$ and iNOS using immunoblotting. (b) The content of nitrate in the CM collected from infected N9 cells was measured using Griess reagent. (c and d) The content of IL-6 and TNF- $\alpha$ in the CM was measured using ELISA. (e and $\mathbf{f}$ ) The protein level of $\mathrm{p}-\mathrm{NF} \kappa \mathrm{B}$ was determined using immunoblotting. The density of the bands was analyzed by the Image $\mathrm{J}$ software and expressed as fold of control. ${ }^{\star} P<0.05,{ }^{* * *} P<0.001$

the addition of L-arginine and found that the transportation of L-arginine into cells was not affected by Jmjd3 suppression (Supplementary Figure S4). Moreover, overexpression of Arginase1 in the kdJmjd3 cells was sufficient to metabolize the extra L-arginine and keep NO production at a low level (Supplementary Figure S4).

To determine whether NO production controlled by the Jmjd3-Arginase1 axis contributes to neuron death, we treated kdJmjd3-N9 cells with various iNOS inhibitors such as L-NAME, L-NMMA and SMT for $24 \mathrm{~h}$. CM was then collected from N9 cell cultures and added to N2A neuron culture. We observed that culture with kdJmjd3-CM caused significant neuron death, which can be attenuated by the treatment with three iNOS inhibitors or Arginase1 overexpression (Figure 5c). To directly observe the microgliainduced neuron death, we cocultured N9 cells with EGFP stable-transfected N2A neurons at a ratio of 10:1. We found that the coculture with kdJmjd3- or kdArginase1-N9 cells led to a significant loss of N2A neurons, whereas Arginase1 overexpression in the kdJmjd3 cells could rescue it (Figures $5 d-g)$. Moreover, the survived N2A neurons showed truncated axons reminiscent of apoptotic process involved (Figures 5h-k). In summary, Arginase 1 could partly alleviate the detrimental effects of increased NO production upon Jmjd3 suppression, and it may have a role in neuroprotection through counteracting iNOS.
Jmjd3 knockdown exacerbates neuron loss in MPTP-intoxicated mouse model. To investigate whether Jmjd3 suppression also produces detrimental effects to DA neurons in vivo, we knocked down Jmjd3 expression by stereotaxic microinjection of lentiviral particles (pLVX-kdNC and pLVX-kdJmjd3) into the left and right sides of the SNc region, respectively, and treated the mice with 1-methyl-4phenyl-1, 2, 3, 6-tetrahydropyridine (MPTP) 1 week later (Figures 6a and b).

By performing immunostaining with anti-tyrosine hydroxylase (TH), we found Jmjd3 knockdown led to greater DA neuron death by $\sim 60 \%$, compared with $\sim 40 \%$ in pLVX-kdNC-injected side of the MPTP-treated mice (Figures $6 \mathrm{c}$ and d). The knockdown of Jmjd3 in saline-injected mice, however, could not cause DA neuron death (Figures $6 \mathrm{c}$ and d). Similarly, immunoblotting assay also showed that the protein level of $\mathrm{TH}$ in the striatum was greatly decreased upon Jmjd3 knockdown after MPTP administration (Figures $6 \mathrm{e}$ and f), suggesting that Jmjd3 knockdown accelerates DA neuron loss in vivo.

Administration of MPTP damaged the DA neurons and induced microglial activation. ${ }^{28}$ As seen in our study, MPTP injection led to extensive microglial overactivation in both $\mathrm{SN}$ and striatum (Supplementary Figure S5). Interestingly, we found that upon Jmjd3 knockdown, a larger population of overactivated microglia with ameboid morphology surrounded 
a

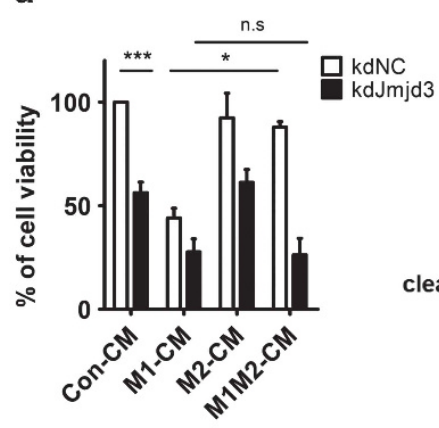

b

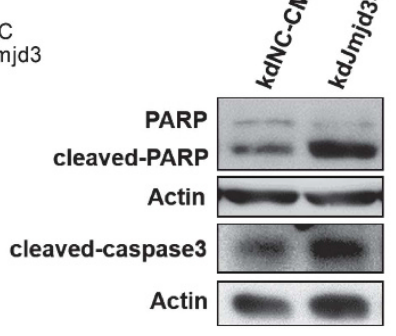

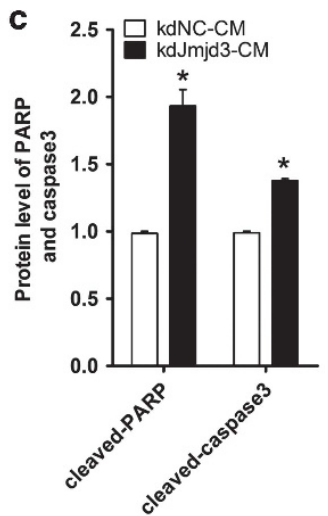

d kdNC-CM
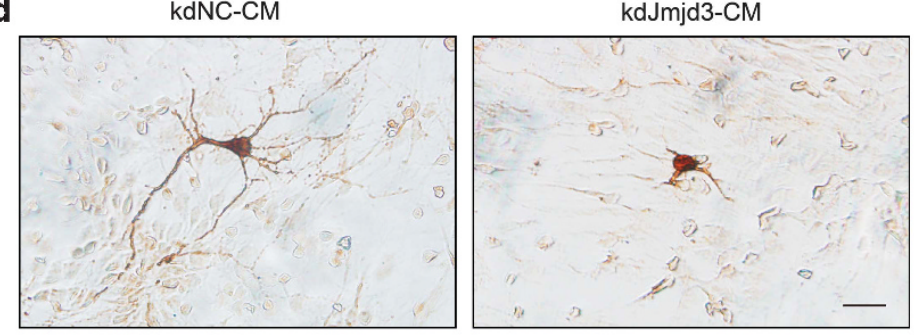

e

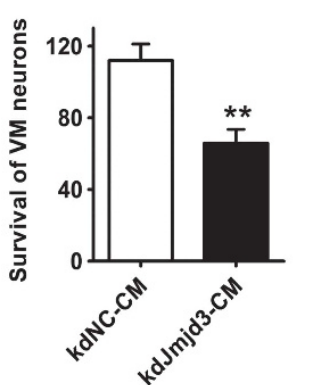

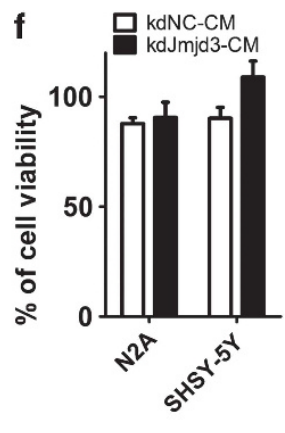

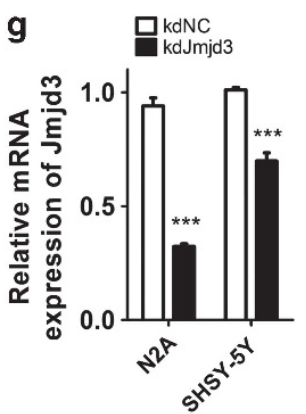

Figure 4 Suppression of Jmjd3 in microglia is detrimental to cultured neurons. (a) The control and Jmjd3-knockdown N9 cells were treated with M1 triggers, M2 triggers and M1/M2 combined triggers, respectively, for $24 \mathrm{~h}$. CMs were then harvested and added into N2A neuron culture for another $24 \mathrm{~h}$. The cell viability of N2A neurons was then assessed using the MTT assay. ( $b$ and $\mathbf{c}$ ) Immunoblotting was then performed to assess the protein level of cleaved caspase 3 and cleaved PARP in the cultured N2A neurons. The density of the bands was analyzed using the Image $\mathrm{J}$ software and expressed as fold of control. (d and e) Primary VM neurons were cultured with kdNC-CM and kdJmid3-CM for $24 \mathrm{~h}$. DA neurons were then visualized using immunostaining with anti-TH antibody (d) and the survival of VM neurons was assessed using the MTT assay (e). Scale bar, $40 \mu \mathrm{m}$. (f) Jmjd3 was knocked down in N2A or SHSY-5Y neurons and CMs were collected and added into N2A neuron culture for $24 \mathrm{~h}$. The cell viability of N2A neurons was then assessed using the MTT assay. (g) The mRNA level of Jmjd3 in the transfected N2A and SHSY-5Y neurons was examined using real-time PCR. n.s, not significant, ${ }^{\star} P<0.05,{ }^{* *} P<0.01,{ }^{* \star *} P<0.001$

DA neurons in the SNc (Figures $6 \mathrm{~g}$ and $\mathrm{h}$ ). However, this overactivation could not lead to neuron death unless MPTP was injected (Figure 6g). It implies that microglial overactivation upon Jmjd3 suppression is more likely an amplifier, which requests MPTP as an initiator, to kill DA neurons. Taken together, these results indicate that Jmjd3 knockdown in vivo can accelerate DA neuron degeneration in the MPTP mouse model by revoking excessive microglial overactivation.

Aging might contribute to the switch of microglia phenotypes from M2 to M1. Increasing evidence has shown that epigenetic mechanisms are involved in regulating aging-related cellular behaviors. ${ }^{29,30}$ It is proposed that the epigenetic modulated switch of microglia polarization is influenced by aging. Indeed, by comparing the mRNA levels of those markers and related molecules in aged mice (16-18 months old) with young mice (2-3 months old), we showed that the M1 markers such as iNOS, TNF- $\alpha$ and IL- 6 were generally increased in aged mice, whereas the M2 markers including Arginase1, CD206 and IGF-I were expressed at lower levels (Figures $7 a$ and b). The neurotrophic factors including brain-derived neurotrophic factor (BDNF) and glial cell-derived neurotrophic factor (GDNF) were determined in aged mice, showing that the BDNF level was significantly reduced, whereas the GDNF level was not changed (Figure 7c).

The increased ratio of $\mathrm{M} 1 / \mathrm{M} 2$ markers might be associated with Jmjd3 modulation. Therefore, we determined the mRNA levels of modified enzymes of histone H3K27me3 including Jmjd3, UTX and the enhancer of zeste homolog 2 (Ezh2). 

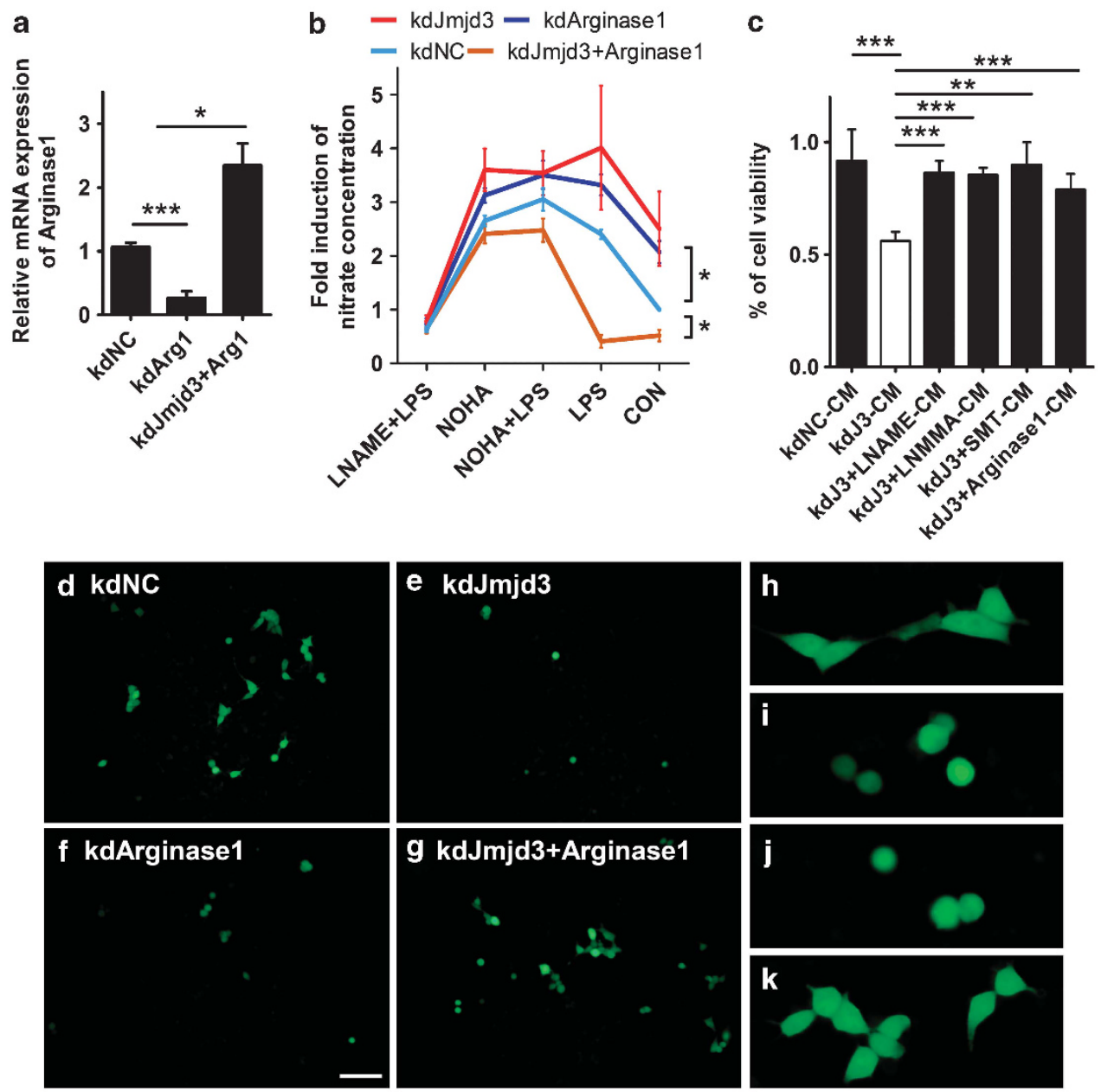

Figure 5 Arginase1 prevents neuron death through counteracting iNOS. (a) N9 cells were infected with pLKO-kdNC, pLKO-kdJmjd3, pLKO-kdArginase1 lentiviruses, as well as pLKO-kdJmjd3/pLVX-Arginase1 mixed lentiviruses. Real-time PCR was then performed to examine the mRNA level of Arginase 1 in the infected cells. (b) Infected cells were pretreated for $4 \mathrm{~h}$ with nor-NOHA $(100 \mu \mathrm{M})$ and L-NAME $(100 \mu \mathrm{M})$ before LPS $(100 \mathrm{ng} / \mathrm{ml})$ treatment for another $24 \mathrm{~h}$. The content of nitrate in the CM was measured using Griess reagent. (c) Lentivirus-infected N9 cells were treated with different iNOS inhibitors including L-NAME (100 $\mu \mathrm{M})$, L-NMMA (100 $\mu \mathrm{M})$ and SMT (200 $\mu \mathrm{M})$ for $24 \mathrm{~h}$. CMs were then harvested and added into N2A neuron culture and the cell viability of N2A neurons was assessed using the MTT assay. (d-g) The EGFP stabletransfected N2A neurons were cocultured in a ratio of $1: 10$ with lentivirus-infected N9 cells in the IMDM complete medium. Twenty-four hours later, the morphology of N2A-EGFP neurons was visualized under microscopy. Scale bar, $100 \mu \mathrm{m}$. (h-k) The morphology of N2A-EGFP neurons was observed in threefold magnification corresponding to $(\mathbf{d}-\mathbf{g}) .{ }^{*} P<0.05,{ }^{* *} P<0.01,{ }^{* * *} P<0.001$

We found that Jmjd3 expression was significantly reduced in aged mice (Figure $7 \mathrm{~d} ; P=0.023$ ). Additionally, the level of UTX was also decreased; however, the level of Ezh2 that governs the methylation of histone H3K27me3 was not altered (Figure $7 \mathrm{~d}$ ). The unbalanced expression of demethylase and methylase was also reflected by the increased H3K27me3 level in the aged midbrain (Figures $7 e$ and $\mathrm{f}$, Supplementary Figure S6). Overall, these results demonstrate that aging contributes to an elevated level of H3K27me3 in the midbrain, which may facilitate the switch of microglia phenotypes from M2 to M1.

\section{Discussion}

Here, we present the evidence that histone H3K27me3 demethylase Jmjd3 is crucial for M2 microglia polarization depending on its demethylase activity. Jmjd3 suppression switches microglia from the beneficial M2 phenotype into the detrimental M1 phenotype and finally accelerates the DA neuron death (Figure 8).

Histone H3K27me3 demethylase Jmjd3 is involved in the neural development and inflammation. ${ }^{21,31,32}$ In our study, we found that Jmjd3 was required and sufficient for M2 microglia polarization. Previously, Satoh et al. ${ }^{33}$ reported that Jmjd3 was necessary for macrophages to mediate alternative activation-like responses against helminth infection and chitin. In his study, the Jmjd3 $3^{-1-}$ bone marrow-derived macrophages (BMMs) were able to upregulate the gene expression of M2 macrophages in response to IL-4 stimulation, suggesting that Jmjd3-mediated H3K27 demethylation is dispensable for the generation of M2 macrophage. ${ }^{33}$ Several other studies have also shown that M1 classical responses and NO production do not differ between wild-type and Jmjd3-deficient macrophages. ${ }^{33,34}$ However, our findings have revealed that 
a

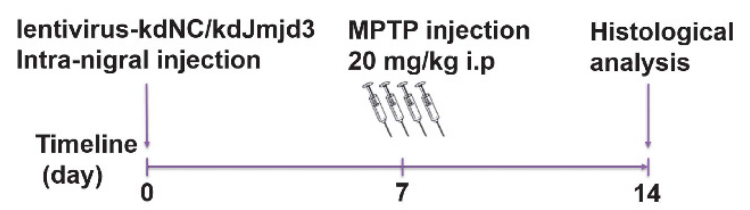

C

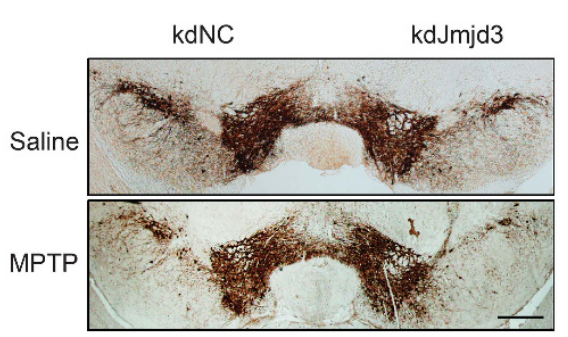

d

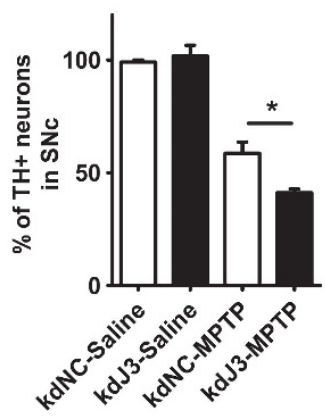

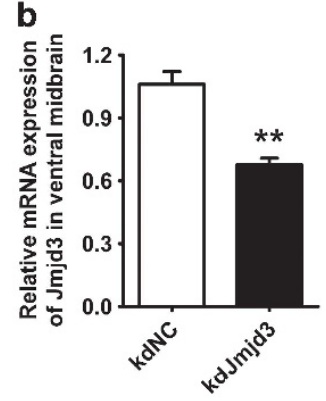

e $\mathrm{TH}$ $\beta$-actin

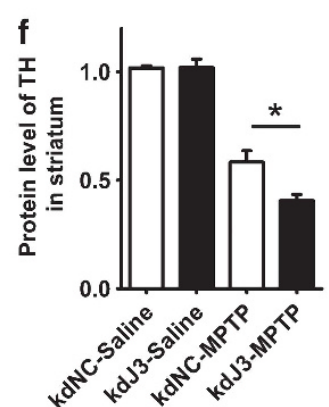

g
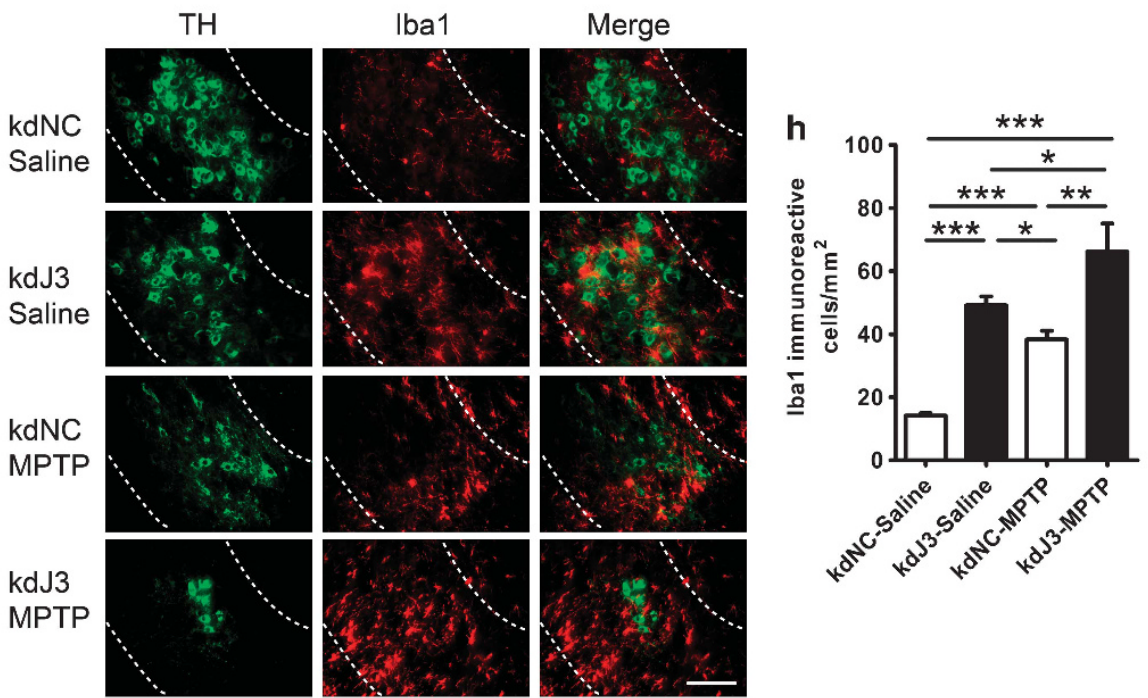

Figure 6 Knockdown of Jmjd3 exacerbates neuron loss in the MPTP-intoxicated mouse model. (a) Schematic representation of the mouse model establishment and lentivirus injection. Lentiviruses (pLVX-kdNC and pLVX-kdJmjd3) were stereologically injected into the left and right side of SNc region, respectively. One week later, MPTP $(20 \mathrm{mg} / \mathrm{kg}$, i.p.) was injected for four times at 2-h intervals to generate the intoxicated model. Mice were then killed and perfused for histological analysis after another week. Total RNA was isolated from dissected ventral midbrain and the protein was harvested from dissected striatum. (b) The mRNA expression of Jmjd3 in the midbrain of injected mice $(n=5)$ was examined using real-time PCR. (c and d) DA neurons in the SNc were visualized using immunostaining with the anti-TH antibody. The number of TH + neurons was counted using a stereological method. Saline group $(n=3)$, MPTP group $(n=6)$. Scale bar, $400 \mu \mathrm{m}$. (e and f) Immunoblotting was performed to assess the protein level of TH in the striatum. Saline group $(n=4)$, MPTP group $(n=5)$. (g) Microglial activation in the SNc (sketched by dash lines) was visualized using doubleimmunostaining with anti-TH and anti-ionized calcium-binding adapter molecule 1 (lba1) antibodies. Scale bar, $200 \mu \mathrm{m}$. (h) Quantification of Iba1 immunoreactive cells in the SNc. ${ }^{*} P<0.05,{ }^{* *} P<0.01,{ }^{* \star *} P<0.001$

Jmjd3-induced M2 microglia polarization is dependent on its demethylation activity. Suppression of Jmjd3 could give rise to exaggerated M1 microglia polarization and promote the production of various pro-inflammatory factors including iNOS, IL-1 $\beta$ and IL-6. These divergent results might be partly because of the differences in the membranous and biochemical markers between peripheral macrophages and microglia, considering microglia is specified in the central nervous system (CNS) microenvironment. ${ }^{35}$ Moreover, it is highly possible that in the $\mathrm{Jmjd} 3^{-1-}$ BMMs, the compensentary mechanisms may interfere the H3K27me3-involved gene regulation. 


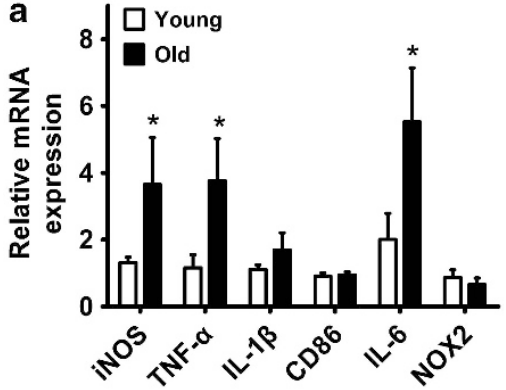

c

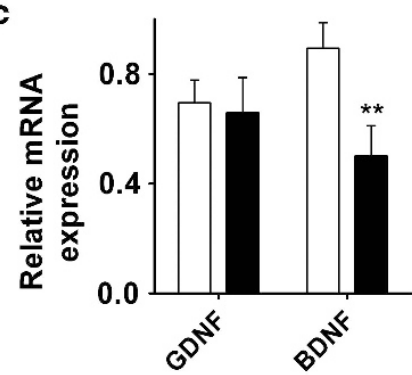

e

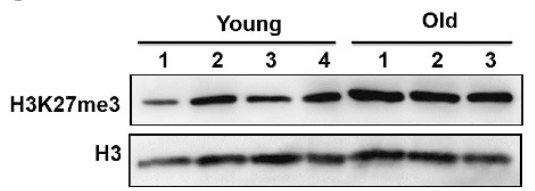

d
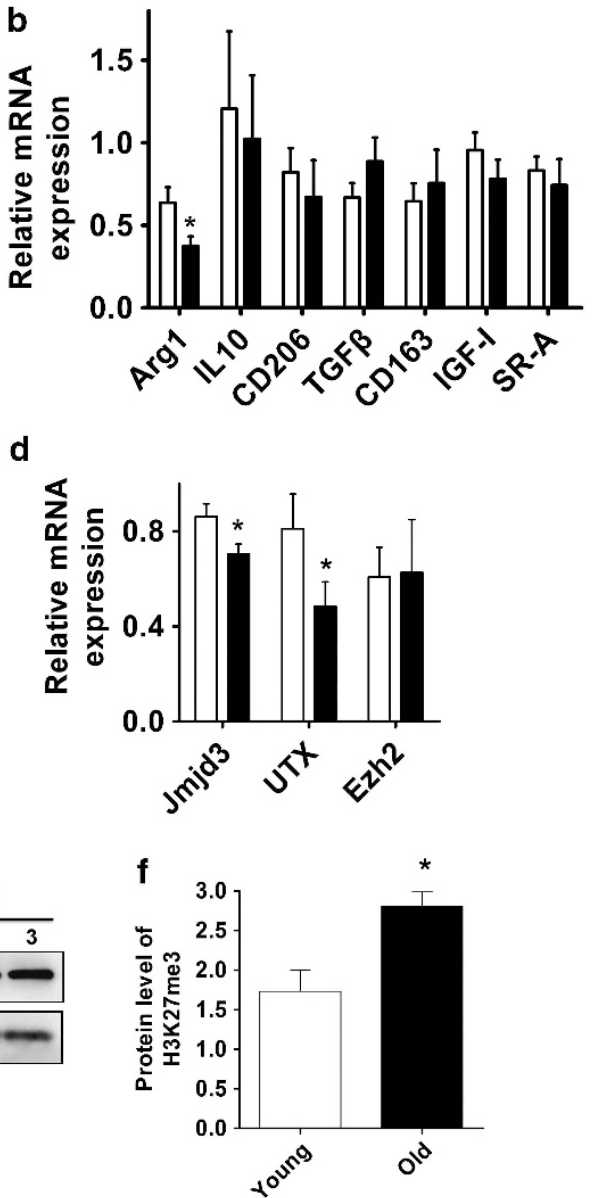

Figure 7 Aging contributes to an elevated level of $\mathrm{H} 3 \mathrm{~K} 27 \mathrm{me} 3$ and an increased ratio of M1/M2 markers in the ventral midbrain. (a-d) Young mice (2-3 months old, $n=8$ ) and old mice (16-18 months old, $n=7$ ) were killed and their ventral midbrains were dissected for mRNA and protein isolation. Real-time PCR was then performed to determine the mRNA level of M1 markers (a), M2 markers (b), neurotropic factors GDNF and BDNF (c) and histone H3K27me3-modified enzymes (d). (e and f) The H3K27me3 level in the midbrain of young (2-3 months old, $n=4)$ and old mice (16-18 months old, $n=3$ ) was assessed using immunoblotting. The density of the bands was analyzed using the Image $\mathrm{J}$ software and expressed as fold of control. ${ }^{*} P<0.05,{ }^{*} P<0.01$

Interestingly, knockdown of Jmjd3 in the microglia even without any treatment compromised the expression of various M2 genes, suggesting that the mechanism of Jmjd3-regulated M2 polarization is very intrinsic and cell-autonomous. Among those targets regulated by Jmjd3, Arginase1 is a typical M2 marker involved in the repair of tissue injury. ${ }^{5}$ Previous studies have shown that Arginase1 can serve as an unexpected beneficial role of Arginase1 in inflammatory diseases such as atherosclerosis. ${ }^{36}$ However, the anti-inflammatory effect of Arginase 1 in the CNS has not been reported. We demonstrate that the suppression of either Jmjd3 or Arginase1 can enhance NO production in a similar manner; however, introduction of full-length Arginase1 into Jmjd3-knockdown cells could suppress the NO elevation and attenuate neuron death. These results confirm that Arginase1 as a downstream target of Jmjd3 could execute anti-inflammatory effects directly by counteracting iNOS. To the best of our knowledge, this is the first study on the neuroprotective functions of Arginase1 in PD pathogenesis. It should be emphasized that M2 polarization is a complex process consisting of many subtypes, in which various downstream targets regulated by Jmjd3 should be further specified.
Furthermore, we examined the role of Jmjd3 in vivo and found that Jmjd3 knockdown in the SN exacerbates DA neuron loss in MPTP-intoxicated mice. The suppression of Jmjd3 induces excessive microglial overactivation. We assume that the DA neuron death induced by MPTP administration initiates a primary inflammation response, which is amplified afterwards by preceded overactivated microglia upon Jmjd3 suppression. The unbalanced M1/2 microglia might contribute to the accelerated neuron death. Generally, the resolution of inflammation and tissue repair mediated by M2 microglia usually occur after the acute phase of inflammation. However, the MPTP-injected mouse is an acute model of DA neuron injury, and it might be advisable to examine the long-term dynamics of M1 and M2 polarization in a chronic inflammatory animal model such as lactacystininduced ubiquitin-proteasome system (UPS)-impairment model. ${ }^{37}$ Moreover, it would be interesting to monitor the balance of microglia polarization in the microglia-specific Jmjd3-knockout mice during the generation of PD.

Aging is one of the most important risk factors for the development and progression of PD. ${ }^{38}$ Many lines of evidence have shown that aged microglia may give rise to altered 

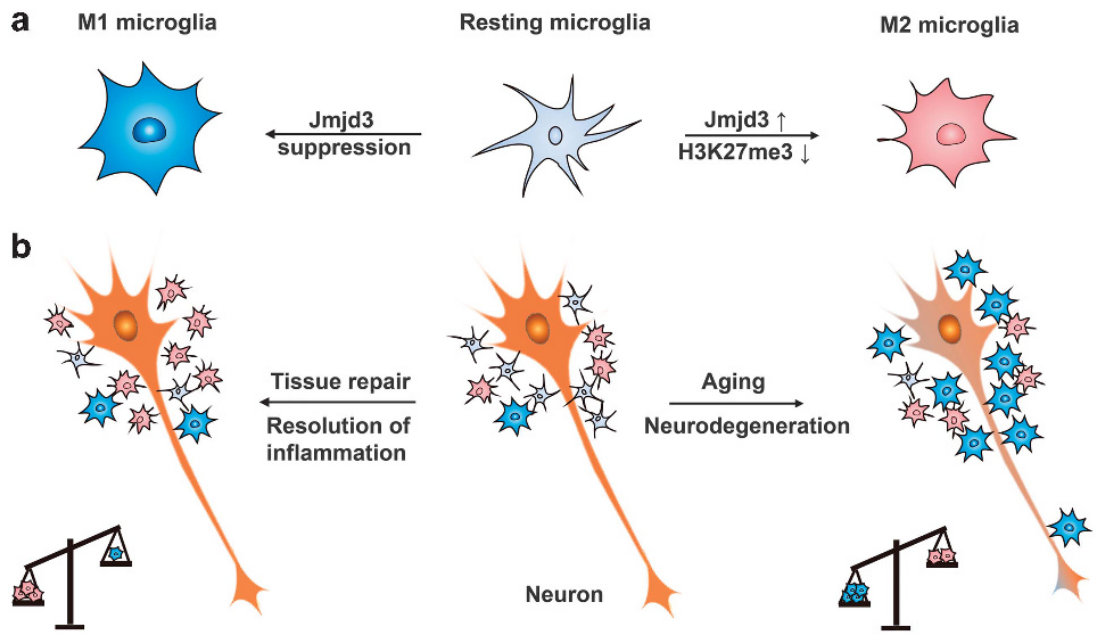

Figure 8 Proposed model of Jmjd3 involved in epigenetic regulation of microglia phenotypes. (a) Under physiological conditions, microglia exist in a resting state with ramified morphology. They dramatically polarize into M1 or M2 phenotype upon different immunological stimuli or injury. Histone H3K27me3 demethylase Jmjd3 is essential for the M2 microglia polarization accompanying with the decreased H3K27me3 level. Suppression of Jmjd3 inhibits M2 polarization and exaggerates M1 polarization. (b) Suppression of Jmjd3 compromises neuroprotective effects of M2 microglia and accordingly exaggerates detrimental effects of M1 microglia. The increased ratio of M1 to M2 microglia contributes to extensive neuron death and aggravates neurodegeneration. Moreover, Jmjd3 is downregulated in the midbrain of aged mice, accompanied by an elevated level of $\mathrm{H} 3 \mathrm{~K} 27 \mathrm{me} 3$ and an increased ratio of M1 to M2 markers, suggesting that aging is a potential factor to switch microglia phenotypes from M2 to M1 that may contribute to the pathogenesis of PD

inflammatory profiles with increased production of pro-inflammatory factors. ${ }^{39-42}$ In aged mice as compared with young mice, treatment with MPTP can cause severe DA neuron loss and greater microglial activation in the SN. ${ }^{43}$ It is possible that aged microglia is undergoing phenotype transformation by epigenetic modification. In our study, we found that the H3K27me3 level was higher, and the levels of Jmjd3 and UTX were lower in the midbrain of aged mice than young controls. Besides, we documented that various M1 markers such as iNOS, TNF- $\alpha$ and IL-6 were increased, whereas the M2 marker Arginase1 was repressed. These results give us a hint that aging might switch microglia phenotypes from $\mathrm{M} 2$ to $\mathrm{M} 1$, and thus aging brains are more easily affected by genetic or environmental insults during the onset of PD (Figure 8). Thus, it might be of interest to know whether the upregulation of the Jmjd3 level in the aging microglia may help to switch its phenotypes and facilitate the resolution of inflammation. Overall, this study may provide new information to address the underlying mechanisms by which the sustained pro-inflammation overrides the beneficial anti-inflammatory effects of microglia, leading to the chronic progression of PD.

\footnotetext{
Materials and Methods

Plasmids and clones. siRNA oligos targeting Jmjd3 (NM_001017426) or Arginase1 (NM_007482) were annealed and inserted into the restriction sites of the pLKO.1 lentiviral vector. The siRNA target sequences are as follows: Jmjd3 (5'-GCTGGATGAATCCATTCGGAA-3'), Arginase 1 (5'-GCTCCTTTCAGGACTA GAT- $\left.3^{\prime}\right)$ and the negative control (NC) sequence $\left(5^{\prime}\right.$-TTCTCCGAACGTGT CACGT-3'). For mice injection, the same siRNA oligos were cloned into pLVXshRNA2 lentiviral vector. The CDNA of the JmjC domain was amplified using the primers (forward primer: $5^{\prime}$-GGCACTAGTATGCGTGCCAGCAAGAATGCC-3' and reverse primer: $5^{\prime}$-GGCGGATCCTCATCGAGACGTGCTGGCGG-3') and was then cloned into the pLVX-IRES-puro lentiviral vector. The $\mathrm{Jmj} \mathrm{Cm}$ construct was generated by Dpnl-mediated site-directed mutagenesis using the mutagenic primers (forward: 5'-AGCCGAACGCCAGGCGCCCAAGAGAATAACAATTTC-3' and reverse: $5^{\prime}$-GAAATTGTTATTCTCTTGGGCGCCTGGCGTTCGGCT-3').
}

Full-length CDNA of Arginase1 or EGFP was cloned into the pLVX-IRES-puro lentiviral vector using the similar strategy.

Cell cultures and treatments. Murine microglial N9 cells were maintained in IMDM medium (Gibco, Rockville, MD, USA) containing $5 \%$ fetal bovine serum (FBS, HyClone, Logan, UT, USA) and 1\% penicillin/streptomycin (PS, Gibco) at $37^{\circ} \mathrm{C}$ in a humidified, $5 \% \mathrm{CO}_{2}$ incubator. Macrophage RAW264.7 cells, mouse N2A neurons and human SHSY-5Y neurons were cultured in DMEM medium (HyClone) supplemented with 10\% FBS and 1\% PS. Until subconfluency, cells were cultured in the medium in the absence of FBS and were insulted with various cytokines including LPS (100 ng/ml, Sigma, St. Louis, MO, USA), recombinant murine IFN $\gamma(10 \mathrm{ng} / \mathrm{ml}$, Peprotech, Rocky Hill, NJ, USA), IL-10 $(10 \mathrm{ng} / \mathrm{ml}$, Peprotech), IL-4 (10 ng/ml, Peprotech) and IL-13 (10 ng/ml, Peprotech) for $24 \mathrm{~h}$. In some cases, cells were pretreated for $4 \mathrm{~h}$ with iNOS inhibitors including L-NAME $(100 \mu \mathrm{M}$, Cayman, Ann Arbor, MI, USA), L-NMMA (100 $\mu \mathrm{M}$, Beyotime, JiangSu, China) and SMT (200 $\mu \mathrm{M}$, Beyotime), Arginase1 inhibitor nor-NOHA (100 $\mu \mathrm{M}$, Cayman) and L-arginine (ranging from 0.5 to $5 \mathrm{mM}$, Beyotime) before cytokine stimulation. Stable-transfected N9 or N2A cells were generated either through infection with lentiviruses or through transfection with lipofectamine 2000 (Invitrogen, Carlsbad, CA, USA), followed by puromycin or G418 selection. Coculture experiments were carried out by plating N9 cells and N2A-EGFP neurons at a ratio of 10:1 and maintained in IMDM medium before observation under the fluorescence microscopy (Olympus IX81, Tokyo, Japan).

CM preparation, nitrate measurement and ELISA. CM was collected at desired time points after cell treatment, filtered through $0.45-\mu \mathrm{m}$ filters and frozen at $-80^{\circ} \mathrm{C}$ for culture with neurons. The production of NO was determined by measuring the accumulated level of nitrate (an indicator of $\mathrm{NO}$ ) in the $\mathrm{CM}$ using a colorimetric reaction with Griess reagent (Beyotime). Briefly, $50 \mu \mathrm{l}$ of $\mathrm{CM}$ was incubated with $50 \mu$ l Griess Reagent I and subsequently $50 \mu$ l Griess Reagent II for $5 \mathrm{~min}$ in the 96-well plate. Optical density (OD) at $540 \mathrm{~nm}$ was then detected and nitrate concentration was calculated according to a $\mathrm{NaNO}_{2}$ standard curve. The concentration of IL- 6 and TNF- $\alpha$ in the CM was determined using the ELISA kit (Westang, Shanghai, China) according to the manufacturer's instructions.

MTT assay. For the cell proliferation assay, N9 cells were plated in a 96-well plate at a density of $1 \times 10^{3}$ cells/well. For the cytotoxicity assay, N2A neurons were cultured at a density of $5 \times 10^{3}$ cells/well for overnight before changing into CM. At desired time points, $20 \mu \mathrm{l}$ of MTT (Sangon, Shanghai, China) was added into the wells and the plate was incubated for $2-4 \mathrm{~h}$ at $37^{\circ} \mathrm{C}$. After that, the medium was discarded and the yielding purple MTT formazan crystals were 
dissolved in DMSO solvent. ODs at 570 and $630 \mathrm{~nm}$ were then measured to determine the cell viability or proliferation ability.

Animals and surgery. C57BL/6 mice (male, 12 weeks old, Shanghai Slac Laboratory) were housed under conditions of constant temperature $\left(20-22{ }^{\circ} \mathrm{C}\right)$ and controlled light $(12 \mathrm{~h} / 12 \mathrm{~h}$ light/dark cycle). Animal care and procedures were carried out in accordance with the Laboratory Animal Care Guidelines approved by the animal committee of Shanghai Institutes for Biological Sciences, Chinese Academy of Sciences.

Mice were anesthetized and placed in a stereotaxic frame and lentiviruses were delivered into the midbrain SN region (AP: $-3.3 \mathrm{~mm}, \mathrm{ML}: \pm 1.2 \mathrm{~mm}$, DV: $-4.6 \mathrm{~mm}$ from bregma). One week later, the mice were injected with MPTP (Sigma, $20 \mathrm{mg} / \mathrm{kg}$ i.p. injection) for four times at 2-h intervals. Mice were killed 1 week after the last MPTP injection. For biochemical assays, mice were killed by decapitation and ventral midbrains/striatum were dissected for total RNA and protein isolation.

For histological analysis, mice were anesthetized and perfused transcardially with cold PBS and 4\% PFA. The whole brains were post-fixed with 4\% PFA overnight and equilibrated in 20 and $30 \%$ sucrose. The brain tissues were then embedded into Tissue-Tek optimal cutting temperature compound (O.C.T., Sakura Finetek Inc., Japan), snap-frozen and stored at $-80^{\circ} \mathrm{C}$. Coronal sections of $10 \mu \mathrm{m}$ were prepared with a Leica cryostat and mounted on PDL-coated slides.

Gene expression. Total RNA was extracted from homogenized brain samples or cultured cells with TRIzol reagent (Invitrogen) according to the manufacturer's instructions. Two micrograms of total RNA were used for cDNA synthesis using ReverTra Ace qPCR RT Kit (Toyobo, Osaka, Japan). Real-time PCR was then performed using SYBR premix Ex TaqTM II kit (Takara, Shiga, Japan) and monitored using the Real-time PCR System (ABI 7500). The primers were verified using melting curve analyses containing a single melt curve peak. The sequences of primers used are provided upon request. The relative expression levels of each mRNA were calculated using the $2^{-\Delta \Delta C t}$ algorithm normalizing to $\beta$-actin and relative to the control samples.

Immunoblotting and immunostaining. See Online Supplementary Materials and Methods.

Stereology. Every fourth section was selected from each animal and processed for immunostaining for $\mathrm{TH}$. The cell number of $\mathrm{TH}$-immunoreactive neurons in the SNc was counted using a stereological method. ${ }^{44}$

Statistical analysis. Results were presented as the mean \pm S.E.M. values. Statistical significance was determined using Student's t-test by GraphPad Prism. The results were considered significant when $P$-value was less than 0.05 .

\section{Conflict of Interest}

The authors declare no conflict of interest.

Acknowledgements. We would like to thank Dr. Yingying Le from the Institute for Nutritional Sciences (INS) of Shanghai Institutes for Biological Sciences (SIBS) for kindly providing N9 cells. This study was supported by grants from the National Natural Sciences Foundation of China (No. 81171201) and the National Basic Research Program of China (No. 2011CB510003).

\section{Author Contributions}

YT and $\mathrm{WL}$ designed the study. YT, TL, JL, JY, HL and XJZ performed the experiments. YT wrote the draft of the manuscript. WL supervised the study and edited the manuscript.

1. Dauer W, Przedborski S. Parkinson's disease: mechanisms and models. Neuron 2003; 39 889-909.

2. Braak H, Del Tredici K, Rub U, de Vos RA, Jansen Steur EN, Braak E. Staging of brain pathology related to sporadic Parkinson's disease. Neurobiol Aging 2003; 24 : 197-211.

3. Imamura K, Hishikawa N, Sawada M, Nagatsu T, Yoshida M, Hashizume Y. Distribution of major histocompatibility complex class II-positive microglia and cytokine profile of Parkinson's disease brains. Acta Neuropathol 2003; 106: 518-526.
4. Hanisch UK, Kettenmann H. Microglia: active sensor and versatile effector cells in the normal and pathologic brain. Nat Neurosci 2007; 10: 1387-1394.

5. Colton CA. Heterogeneity of microglial activation in the innate immune response in the brain. J Neuroimmune Pharmacol 2009; 4: 399-418.

6. Liu B, Gao HM, Wang JY, Jeohn GH, Cooper CL, Hong JS. Role of nitric oxide in inflammation-mediated neurodegeneration. Ann N Y Acad Sci 2002; 962: 318-331.

7. Le W, Rowe D, Xie W, Ortiz I, He Y, Appel SH. Microglial activation and dopaminergic cell injury: an in vitro model relevant to Parkinson's disease. J Neurosci 2001; 21: 8447-8455.

8. Li R, Huang YG, Fang D, Le WD. (-)-Epigallocatechin gallate inhibits lipopolysaccharideinduced microglial activation and protects against inflammation-mediated dopaminergic neuronal injury. J Neurosci Res 2004; 78: 723-731.

9. Saijo K, Winner B, Carson CT, Collier JG, Boyer L, Rosenfeld MG et al. A Nurr1/CoREST pathway in microglia and astrocytes protects dopaminergic neurons from inflammationinduced death. Cell 2009; 137: 47-59.

10. Mayo L, Stein R. Characterization of LPS and interferon-gamma triggered activationinduced cell death in N9 and primary microglial cells: induction of the mitochondrial gateway by nitric oxide. Cell Death Differ 2007; 14: 183-186.

11. Colton C, Wilcock DM. Assessing activation states in microglia. CNS Neurol Disord Drug Targets 2010; 9: 174-191.

12. Giaume $C$, Kirchhoff F, Matute $C$, Reichenbach A, Verkhratsky A. Glia: the fulcrum of brain diseases. Cell Death Differ 2007; 14: 1324-1335.

13. Block ML, Hong JS. Microglia and inflammation-mediated neurodegeneration: multiple triggers with a common mechanism. Prog Neurobiol 2005; 76: 77-98.

14. Teismann P, Tieu K, Cohen O, Choi DK, Wu DC, Marks D et al. Pathogenic role of glial cells in Parkinson's disease. Mov Disord 2003; 18: 121-129.

15. Magnus T, Chan A, Grauer O, Toyka KV, Gold R. Microglial phagocytosis of apoptotic inflammatory $T$ cells leads to down-regulation of microglial immune activation. $\mathrm{J}$ Immunol 2001; 167: 5004-5010.

16. Liu Y, Hao W, Letiembre M, Walter S, Kulanga M, Neumann $\mathrm{H}$ et al. Suppression of microglial inflammatory activity by myelin phagocytosis: role of p47-PHOX-mediated generation of reactive oxygen species. J Neurosci 2006; 26: 12904-12913.

17. Polazzi E, Contestabile A. Reciprocal interactions between microglia and neurons: from survival to neuropathology. Rev Neurosci 2002; 13: 221-242.

18. Zhang Z, Zhang ZY, Schluesener HJ. Compound A, a plant origin ligand of glucocorticoid receptors, increases regulatory $T$ cells and M2 macrophages to attenuate experimental autoimmune neuritis with reduced side effects. J Immunol 2009; 183: 3081-3091.

19. Berger SL. The complex language of chromatin regulation during transcription. Nature 2007; 447: 407-412.

20. Portela A, Esteller M. Epigenetic modifications and human disease. Nat Biotechnol 2010; 28: 1057-1068.

21. Agger K, Cloos PA, Christensen J, Pasini D, Rose S, Rappsilber J et al. UTX and JMJD3 are histone $\mathrm{H} 3 \mathrm{~K} 27$ demethylases involved in $\mathrm{HOX}$ gene regulation and development. Nature 2007; 449: 731-734.

22. Mantovani A, Sica A, Sozzani S, Allavena P, Vecchi A, Locati M. The chemokine system in diverse forms of macrophage activation and polarization. Trends Immunol 2004; 25: 677-686.

23. Vanden Berghe W, Ndlovu MN, Hoya-Arias R, Dijsselbloem N, Gerlo S, Haegeman G. Keeping up NF-kappaB appearances: epigenetic control of immunity or inflammationtriggered epigenetics. Biochem Pharmacol 2006; 72: 1114-1131.

24. Cho IH, Hong J, Suh EC, Kim JH, Lee H, Lee JE et al. Role of microglial IK Kbeta in kainic acid-induced hippocampal neuronal cell death. Brain 2008; 131: 3019-3033.

25. Aoki $\mathrm{E}$, Yano R, Yokoyama $\mathrm{H}$, Kato $\mathrm{H}$, Araki T. Role of nuclear transcription factor kappa $\mathrm{B}$ (NF-kappaB) for MPTP (1-methyl-4-phenyl-1,2,3,6-tetrahyropyridine)-induced apoptosis in nigral neurons of mice. Exp Mol Pathol 2009; 86: 57-64.

26. Bronte V, Zanovello P. Regulation of immune responses by L-arginine metabolism. Nat Rev Immunol 2005; 5: 641-654.

27. Lee J, Ryu H, Ferrante RJ, Morris SM Jr., Ratan RR. Translational control of inducible nitric oxide synthase expression by arginine can explain the arginine paradox. Proc Natl Acad Sci USA 2003; 100: 4843-4848.

28. Liberatore GT, Jackson-Lewis V, Vukosavic S, Mandir AS, Vila M, McAuliffe WG et al. Inducible nitric oxide synthase stimulates dopaminergic neurodegeneration in the MPTP model of Parkinson disease. Nat Med 1999; 5: 1403-1409.

29. Kuzumaki N, Ikegami D, Tamura R, Sasaki T, Niikura K, Narita M et al. Hippocampal epigenetic modification at the doublecortin gene is involved in the impairment of neurogenesis with aging. Synapse 2010; 64: 611-616.

30. Zeng $Y$, Tan M, Kohyama J, Sneddon M, Watson JB, Sun YE et al. Epigenetic enhancement of BDNF signaling rescues synaptic plasticity in aging. J Neurosci 2011; 31 : $17800-17810$

31. De Santa F, Totaro MG, Prosperini E, Notarbartolo S, Testa G, Natoli G. The histone H3 lysine-27 demethylase Jmjd3 links inflammation to inhibition of polycomb-mediated gene silencing. Cell 2007; 130: 1083-1094.

32. Jepsen K, Solum D, Zhou T, McEvilly RJ, Kim HJ, Glass CK et al. SMRT-mediated repression of an $\mathrm{H} 3 \mathrm{~K} 27$ demethylase in progression from neural stem cell to neuron. Nature 2007; 450: 415-419. 
33. Satoh T, Takeuchi O, Vandenbon A, Yasuda K, Tanaka Y, Kumagai Y et al. The Jmjd3-Irf4 axis regulates $\mathrm{M} 2$ macrophage polarization and host responses against helminth infection. Nat Immunol 2010; 11: 936-944.

34. Ishii M, Wen H, Corsa CA, Liu T, Coelho AL, Allen RM et al. Epigenetic regulation of the alternatively activated macrophage phenotype. Blood 2009; 114: 3244-3254.

35. Guillemin GJ, Brew BJ. Microglia,macrophages, perivascular macrophages, and pericytes: a review of function and identification. J Leukoc Biol 2004; 75: 388-397.

36. Wang XP, Chen YG, Qin WD, Zhang W, Wei SJ, Wang $J$ et al. Arginase I attenuates inflammatory cytokine secretion induced by lipopolysaccharide in vascular smooth muscle cells. Arterioscler Thromb Vasc Biol 2011; 31: 1853-1860.

37. Xie WJ, Li XP, Li C, Zhu W, Jankovic J, Le WD. Proteasome inhibition modeling nigral neuron degeneration in Parkinson's disease. J Neurochem 2010; 115: 188-199.

38. Levy G. The relationship of Parkinson disease with aging. Arch Neurol 2007; 64: 1242-1246.
39. Ye SM, Johnson RW. Increased interleukin- 6 expression by microglia from brain of aged mice. J Neuroimmunol 1999; 93: 139-148.

40. Sierra A, Gottfried-Blackmore AC, McEwen BS, Bulloch K. Microglia derived from aging mice exhibit an altered inflammatory profile. Glia 2007; 55: 412-424.

41. Lee CK, Weindruch R, Prolla TA. Gene-expression profile of the ageing brain in mice Nat Genet 2000; 25: 294-297.

42. Sheng JG, Mrak RE, Griffin WS. Enlarged and phagocytic, but not primed, interleukin-1 alpha-immunoreactive microglia increase with age in normal human brain Acta Neuropathol 1998; 95: 229-234.

43. Sugama S, Yang L, Cho BP, DeGiorgio LA, Lorenzl S, Albers DS et al. Age-related microglial activation in 1-methyl-4-phenyl-1,2,3,6-tetrahydropyridine (MPTP)-induced dopaminergic neurodegeneration in C57BL/6 mice. Brain Res 2003; 964: 288-294.

44. West MJ. New stereological methods for counting neurons. Neurobiol Aging 1993; 14: 275-285.

Supplementary Information accompanies this paper on Cell Death and Differentiation website (http://www.nature.com/cdd) 